\title{
SOFTWARE EDUCATIVO "MUNDO AGROFORESTAL": Estudio de caso, Subcuenca Alta del Río Pasto, Nariño, Colombia
}

\section{AGROFORESTRY EDUCATIONAL SOFTWARE: Case study, Upper Sub-basin of the Pasto River, Nariño, Colombia}

\author{
Ángela Lucía León I. ${ }^{1}$; Gloria Cristina Luna C. ${ }^{2}$; Hugo Ferney Leonel ${ }^{3}$ \\ Fecha de recepción: Abril 28 de 2014 \\ Fecha de aceptación: Junio 9 de 2014
}

\begin{abstract}
RESUMEN
Las Tecnologías de la Información y la Comunicación (TIC), son herramientas que han permitido evolucionar en el proceso de enseñanza aprendizaje, innovación, difusión de información, adquisición y generación de conocimiento. El objetivo de la investigación fue desarrollar un "Software Educativo Agroforestal - SEAf", dirigido a jóvenes de instituciones educativas rurales, como estrategia pedagógica para la enseñanza agroforestal. Mediante Investigación Acción Participativa (IAP), se diagnosticó el conocimiento agroforestal de estudiantes de dos instituciones educativas municipales de la subcuenca alta del río Pasto, implementando talleres participativos, encuestas semi-estructuradas y mapas parlantes; se realizó un análisis de correspondencias múltiples (ACM), que permitió diseñar y estructurar el SEAf con el programa Flash Cs4, el cual fue evaluado con pruebas Alfa y Beta. Como resultado se logró diseñar un software educativo agroforestal denominado "Mundo Agroforestal" que incluyó los gustos y preferencias de los jóvenes; de acuerdo al color, actividades lúdicas, paisajes y personajes animados que fueron plasmados en las interfaces. El software fue altamente aceptado (94\%) respecto a las variables diseño, facilidad de navegación, pertinencia de color, temáticas abordadas e ilustraciones; lo que permitió la validación de éste.
\end{abstract}

Palabras clave: TIC, software, agroforestería, río Pasto.

1 Ingeniera Agroforestal. Facultad de Ciencias Agrícolas. Universidad de Nariño, San Juan de Pasto, Colombia. angelita-leon@gmail.com

2 Docente Tiempo Completo. I.A M.Sc. Facultad de Ciencias Agrícolas. Universidad de Nariño, San Juan de Pasto, Colombia. grupopifil@gmail.com

3 Docente Tiempo Completo. I. F Ph.D. Facultad de Ciencias Agrícolas. Universidad de Nariño, San Juan de Pasto, Colombia. hleonel2001@gmail.com 


\begin{abstract}
Information and Communication Technologies (ICT) are tools that have allowed an evolution in the teaching and learning process, innovation, dissemination of information, and the acquisition and generation of knowledge. The need to implement teaching strategies for agroforestry education led to the development of an "Agroforestry Educational Software" for students of educational institutions in the rural sector. The diagnosis of agroforestry knowledge was evaluated in two institutions of the Pasto River sub-basin, using a Participatory Action Research methodology, by implementing participatory workshops, semi-structured surveys, talking maps, among others; surveys was performed with their respective multiple correspondence analysis (MCA), which allowed the design and structure of the "Agroforestry Software" using a Flash CS4 program for validation and the implementation of Alpha and Beta tests. Limited agroforestry knowledge is evident; however, it is highlighted by the importance of trees on their farms. As for the use of organic fertilizers, this yielded a negative answer, since chemicals inputs are preferred, which led to the need for training in agroforestry principals. The software design highlighted the preference for blue and green colors, competitive activities, landscapes representing forests and beaches, the favorite character was the "Puss in Boots" and animals like dogs and cats; which allowed the creation of a hybrid character. The Alpha and Beta tests showed acceptance of the software in both content and design.
\end{abstract}

Keywords: TIC, software, agroforestry, Pasto River

\section{INTRODUCCIÓN}

Nariño es un departamento de vocación agrícola, caracterizado por el minifundio y explotación inadecuada de los recursos naturales, en especial el suelo y la flora; que en algunos casos, se sobreexplota por las actividades agropecuarias que sobrepasan su capacidad de resilencia; siendo una de las causas la falencia de modelos pedagógicos que conduzcan a la valoración y aprovechamiento adecuado de los recursos naturales e implementación de tecnologías acordes a la realidad socioambiental, cultural y económica; entre ellas la agroforestería y estructuración de herramientas didácticas basadas en Tecnologías de la Información y las Comunicaciones (TIC).

A nivel mundial, para el intercambio de conocimientos agroforestales, se ha implementado las TIC, a partir del diseño de software con este enfoque (académico a nivel superior e investigativo); entre los que se destacan: modelamiento - WaNuLCAS; sistema de manejo agroforestal - Silvia, simulador de bosques - SExl-FS; evaluador de paisajes - FALLOW; generadores de caudal hídrico - SLIM, series temporales de lluvias -SpatRain, entre otros (World Agroforestry Centre, 2011). Sin embargo, existe la falencia de un software más amigable dirigido a jóvenes rurales, que sirva como herramienta pedagógica para la percepción de este conocimiento.

Las TIC, son herramientas que procesan, almacenan, sintetizan, recuperan y presentan información de forma variada (Lavado, 2011); posibilitan la transversalidad en todas las áreas del saber expresando experiencias demostrativas donde los niños se motivan para manejar el lenguaje y desarrollar sus habilidades básicas como: comprender, analizar, sintetizar, hacer referencias y deducciones (Galvis et al., 2012). En este sentido, las TIC, son útiles y contribuyen para la enseñanza aprendizaje de los diferentes temas relacionados con la agroforestería; considerada como una alternativa 
para optimizar los sistemas tradicionales de agricultura y de silvicultura regional y amortizar sus impactos socio-ambientales, orientada a mejorar la calidad de vida de los productores rurales (World Agroforestry Centre, 2011).

Por lo tanto, la presente investigación tuvo como objetivo la creación de una herramienta para facilitar la aprehensión del conocimiento agroforestal. Inició con un proceso diagnóstico trabajando con estudiantes de las Instituciones Educativas Municipales de Mocondino y Cabrera. Para el diseño, se contó con la participación de ingenieros: agrónomo, forestal y agroforestal; diseñador gráfico y licenciado en informática.

El software se caracteriza por contener interfaces amigables y dinámicas, con lenguaje claro que despierta interés en los jóvenes por su innovación; Robey (2007), plantea que la información se puede transmitir de manera más llamativa por diferentes medios, como texto, imagen, sonido, animaciones, entre otros.

Integrar curricularmente las TIC en el proceso de aprendizaje, implica emplearlas de forma creativa, transparente y pertinente en el que hacer pedagógico, y no sólo como una forma alternativa y llamativa de presentar información; es necesario convertirlas en herramientas de apoyo al proceso de aprendizaje; es decir, teniendo siempre en mente que se usan con el objeto de aprender; por tanto, el SEAf "Mundo Agroforestal" está diseñado con fines didácticos, para ser interactivo y fácil de manejar; Marques (2001), explica que la combinación de estos elementos incrementa la capacidad de aprehensión de conocimientos y el interés por parte de los estudiantes; de esta manera, los objetivos propuestos en esta investigación hacen parte de un macro proyecto denominado "Herramientas didácticas para la adopción de sistemas agroforestales en la zona Andina del departamento de Nariño" apoyado por la
Vicerrectoría de Investigaciones Postgrados y Relaciones Internacionales de la Universidad de Nariño, a fin de diagnosticar el conocimiento agroforestal de los estudiantes pertenecientes a las instituciones educativas de Cabrera y Mocondino y con sus contribuciones diseñar y validar un software educativo con enfoque agroforestal.

Se optó por trabajar con jóvenes de instituciones del sector rural, considerando que para el 2005, más de la mitad de la población de Nariño (53\%) vivía en la zona rural; proyectada para el 2010, en una población total de 1.639.569, equivalente al $52 \%$ en el sector rural, se estima que la población de jóvenes rurales desde los 11 hasta los 19 años, asciende a más del 20\% (DANE, 2010); lo que demuestra, la ruralidad del departamento y por ende la agricultura como principal actividad económica; y la necesidad de capacitación de los jóvenes en temas relacionados con el campo, entre ellos: manejo y conservación de recursos naturales y sistemas productivos sustentables como los sistemas agroforestales (SAF).

Así mismo, esta investigación se basó en lineamientos de la apropiación social del conocimiento, entendida, por COLCIENCIAS (2012), como un proceso de comprensión e intervención de las relaciones entre tecnociencia y sociedad, construido a partir de la participación activa de los diversos grupos sociales que generan conocimiento más allá de las sinergias entre sectores académicos, productivos y estatales; integrando apropiación e innovación en un mismo plano.

\section{MATERIALES Y MÉTODOS}

Localización. La investigación se realizó en dos Instituciones Educativas Municipales pertenecientes a la subcuenca alta del Río Pasto, con estudiantes entre los 11 y 19 años de edad. La 
Institución Educativa Municipal Cabrera, localizada en el corregimiento de Cabrera, municipio de Pasto, Nariño, Colombia; ubicada a una altura de 3.000 m.s.n.m, temperatura promedio de $8^{\circ} \mathrm{C}$ y una población de 1.333 habitantes (DANE, 2012) y la Institución Educativa Municipal Mocondino, localizada en el corregimiento de Mocondino, municipio de Pasto, Nariño, Colombia; ubicada a una altura de 2.800 m.s.n.m, temperatura promedio de $14^{\circ} \mathrm{C}$ y una población de 6.800 habitantes aproximadamente. (Ministerio de tecnologías de la información y las comunicaciones de la República de Colombia, 2008). En la tabla 1, se relaciona el número de estudiantes participantes por grado e institución.

De total de los estudiantes (381), el 62\% corresponde a mujeres; en su mayoría, provienen de un núcleo familiar campesino, con una unidad familiar promedia de 5 integrantes; cuya actividad económica principal es la agropecuaria, en unidades de producción mini y microfundistas, que por lo general no superan la hectárea.

El acceso a las TIC es limitado; sin embargo, con el programa "Computadores para Educar" las instituciones cuentan con unidades de PC de escritorio y mejoramiento de infraestructura tecnológica; las cuales, no son suficientes para el número de educandos.

Según la Secretaría Educación Municipal de Pasto (2012), en el sector rural es notable la baja profundización en los conocimientos, desmotivación y poca orientación de las capacidades del estudiante; además, de la falta de pertinencia y relación entre lo que se desea aprender y lo que se ofrece en el establecimiento educativo.

Con el enfoque de Investigación Acción participativa - IAP (Fals, 2008), se evaluó el conocimiento agroforestal de los estudiantes de las Instituciones Educativas Municipales de Cabrera y Mocondino (Tab.1); para ello, se utilizaron varias técnicas y herramientas, como mapas parlantes (visión histórica, presente y futura), a través de los cuales, los estudiantes plasmaron la situación actual de los sistemas productivos de sus fincas y plantearon su deseo de cambio a futuro (cinco años). De igual forma, mediante la lúdica, el diálogo de saberes y la construcción colectiva de maquetas agroforestales, se generaron directrices y pautas de gran importancia, para ser incluidas en el SEAf.

Además, se diseñaron dos encuestas aplicadas al total de la población (381 estudiantes con edades entre 11 y 19 años); en la primera, se evaluó el conocimiento agroforestal a través de 20 preguntas distribuidas en cuatro variables (existencia de sistema productivo, importancia de árboles, participación en labores de finca, conocimiento sobre temáticas agroforestales); en la segunda encuesta, se evaluaron los gustos y preferencias en cinco variables: color, personajes animados, juegos, actividades y paisajes. Se realizó un análisis de correspondencias múltiples (ACM), valores propios y contribución de las variables por medio del software estadístico SPAD V 5.6.

Tabla 1. Relación del número de estudiantes por institución educativa y grado, 2013

\begin{tabular}{l|c|c|c|c|c|c}
\hline \multirow{2}{*}{ Institución Educativa Municipal } & \multicolumn{6}{|c}{ No. de estudiantes por Grado } \\
\cline { 2 - 8 } & $\mathbf{6}$ & $\mathbf{7}$ & $\mathbf{8}$ & $\mathbf{9}$ & $\mathbf{1 0}$ & $\mathbf{1 1}$ \\
\hline Cabrera & 37 & 26 & 26 & 22 & 25 & 19 \\
\hline Mocondino & 43 & 52 & 29 & 36 & 43 & 23 \\
\hline Subtotal & $\mathbf{8 0}$ & $\mathbf{7 8}$ & $\mathbf{5 5}$ & $\mathbf{5 8}$ & $\mathbf{6 8}$ & $\mathbf{4 2}$ \\
\hline Total & & & & & $\mathbf{3 8 1}$ & \\
\hline
\end{tabular}


De acuerdo a las necesidades de capacitación identificadas, se procedió a instruir a los estudiantes sobre conceptos básicos de agroforestería, considerando éste de gran importancia para continuar el proceso de diseño del Software.

Con la información obtenida y haciendo uso del programa de creación y manipulación de gráficos vectoriales FlashCs4, se diseñó el SEAf, con el apoyo de un equipo interdisciplinario, quienes analizaron la información de base y determinaron los componentes del SEAf, considerando contenidos y juegos de evaluación y recreación en relación a definiciones sobre temáticas afines al medio ambiente y agroforestería. De esta manera se definió la estructura del software: programas de ejercitación, bases de datos y galería de imágenes; y los contenidos específicos mediante (video, sonidos, imágenes, texto y animación). Además, se elaboró un manual del usuario para orientar en su instalación y navegación.

Evaluación y validación. Se aplicó una prueba Alfa, con cinco estudiantes seleccionados al azar por cada grado e institución, lo que permitió determinar el nivel de entendimiento y aceptación del SEAf y definir los campos con necesidad de corrección y ajuste. Se efectuó una prueba Beta, empleando una encuesta conformada por nueve preguntas categorizadas en: armonía de interfaces, color, sonido, navegación y diseño; aplicada a una muestra al azar de 20 personas que no hicieron parte de los momentos anteriores, pero que tenían la connotación de ser futuros usuarios del software; quienes fueron los encargados de valorarlo para procesos de aprendizaje.

\section{RESULTADOS Y DISCUSIÓN}

Diagnóstico del conocimiento agroforestal. Se evidenció que en promedio el $95 \%$ de los participantes no tenían conocimiento agroforestal, pero si sobre la importancia del árbol en sus fincas. Los resultados más relevantes fueron:

La situación plasmada en los mapas parlantes, evidencia que los estudiantes identificaron los sistemas de producción actuales, donde prevalecen los monocultivos y potreros desnudos. Mediante un diálogo de saberes, ellos plantearon en el mapa su visión a futuro, donde incluyeron el componente perenne leñoso en forma de arreglos agroforestales; demostrándose la actitud favorable del estudiante para su aprendizaje significativo, es decir, se logró conectar el nuevo conocimiento con el existente, modificando su estructura cognitiva; Ausubel et al. (1983), plantea que el estudiante relaciona el material educativo con la situación nueva de aprendizaje.

Leonel et al. (2013), plantean que los materiales educativos son importantes en el reconocimiento socio-cultural y motivacional; en donde el juego, en especial el tradicional permite revelar a que cultura social pertenece el sujeto y la noción de cohesión social, cobra todo su sentido y espacio; privilegiándose una concepción del mismo sujeto, la cultura, el enseñar y el aprender, sobre: la naturaleza, la comunicación y el sentido de conocimiento; lo anterior, se evidenció cuando los estudiantes conjugaron sus conocimientos en la visión de finca comparándola con la de sus compañeros, observándose amplia comunicación, espacios de análisis y planteamiento de hipótesis que conllevaron a vivencias motivadoras por el tema en cuestión y despertó mayor interés por el aprendizaje agroforestal detallado en los resultados sobre conocimientos relacionados con la agroforestería que manifestaron los estudiantes, Figura 1 (A y B).

Comosemuestra enla figura 1-A, la gran mayoría de estudiantes poseen un sistema productivo en sus fincas; entre los que se destacan los cultivos de: cebolla Allium fistulosum L., papa 


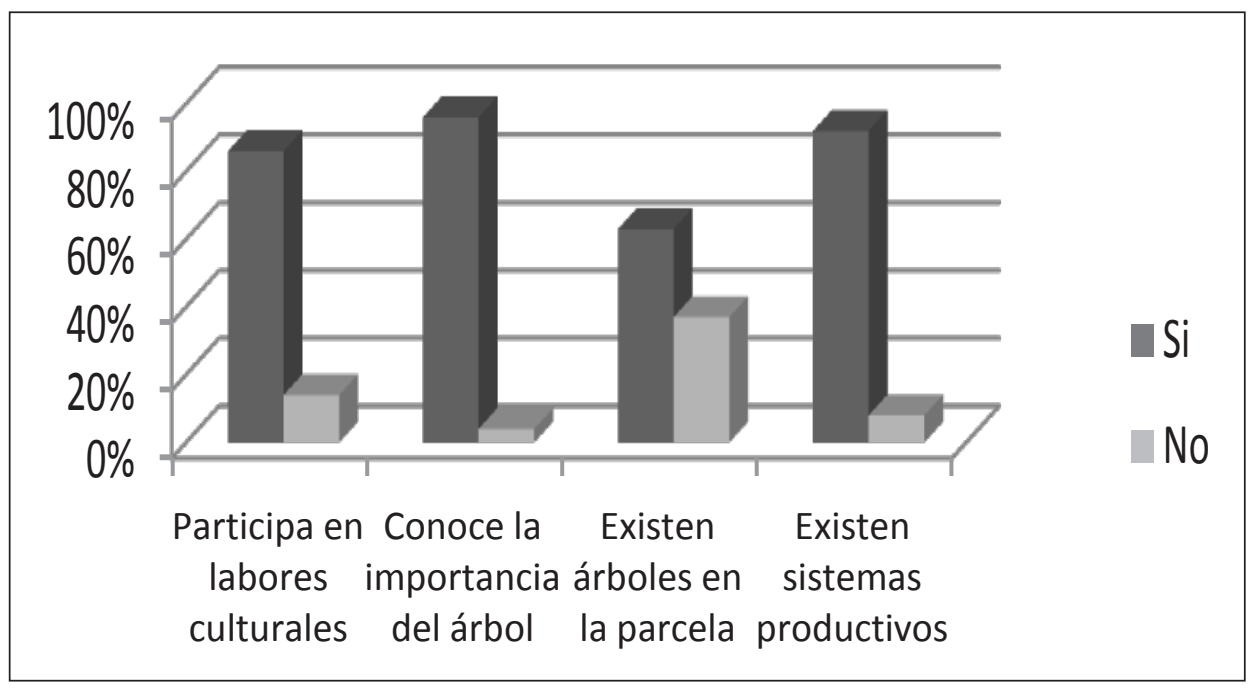

A: Participación y Conocimientos de la importancia del árbol en sistemas productivos

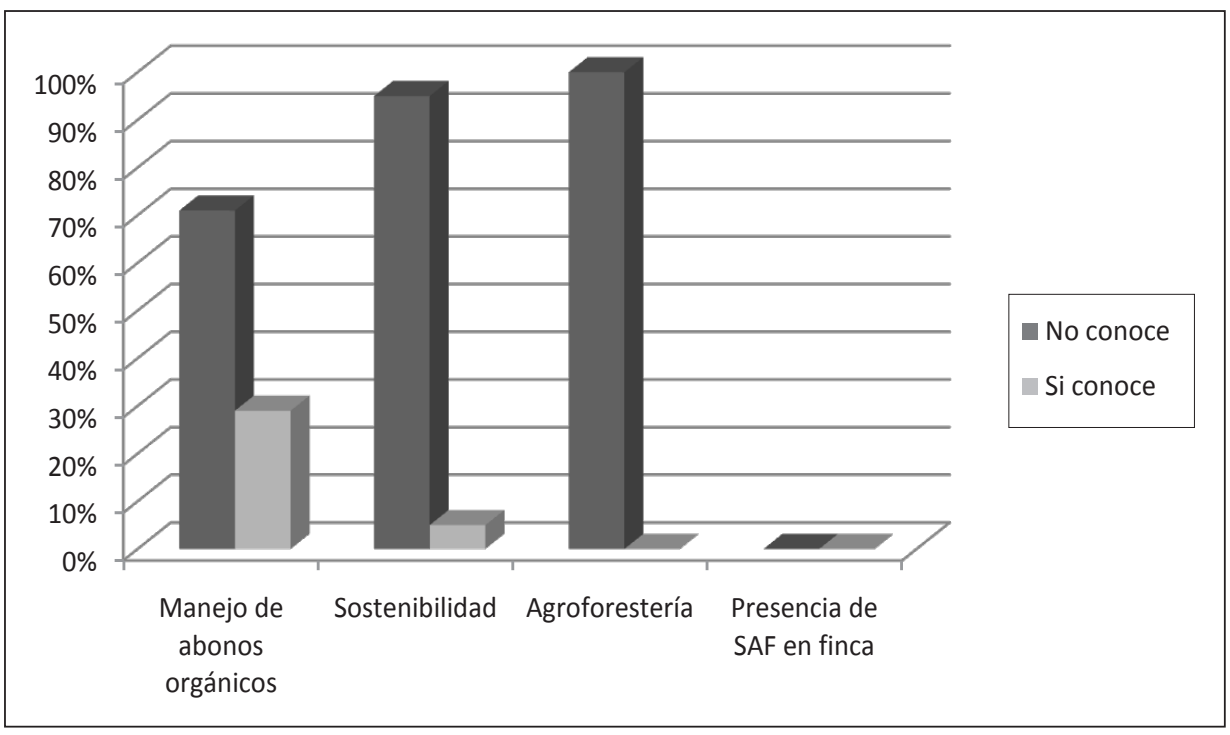

B: Elementos integradores del conocimiento agroforestal

Figura 1. Diagnóstico del conocimiento agroforestal en las instituciones educativas municipales de Cabrera y Mocondino (2013).

Solanum tuberosum L., zanahoria Daucus carota L., remolacha Beta vulgaris L., Repollo Brassica oleracea L., Maíz Zea maíz L., Frijol Phaseolus vulgaris L., Arveja Pisum sativum L., Acelga Beta vulgaris var cicla L., Coliflor Brassica oleracea var botrytis L., Mora Rubus glaucus B., Calabaza Cucurbita máxima D., Brócoli Brassica oleracea var itálica P., Fresa Fragaria vesca C.; Ruda Ruta graveolens L., Manzanilla Matricaria chamomilla
L., Caléndula Calendula officinalis L., Cilantro Coriandrum satioum L.

El Plan de Desarrollo del Municipio de Pasto 2012-2015 "Transformación productiva" (Honorable Concejo Municipal de Pasto. 2012), afirma que su población rural, se caracteriza por tener una economía basada en la producción agropecuaria de pequeños productores. El 87\%, 
son predios con una área promedio de 0,5 a 5 ha, con producción en cultivos de $S$. tuberosum L, especialmente sobre los corregimientos de Santa Bárbara, Catambuco, La Laguna y El Encano; hortalizas A.fistulosum L, Brassica oleracea L, B Brassica oleracea var botrytis L, B. oleracea var itálica $\mathrm{P}$, entre otras; en los corregimientos de Gualmatán, Buesaquillo, Cabrera, Catambuco, San Fernando, Mocondino y Obonuco; cultivos de R. glaucus B., en los corregimientos del Encano y Santa Bárbara; y en La Caldera, Coffea arabica L.

Se observó que en la mayoría de las fincas hay presencia entre dos y tres árboles, destacándose: Eucalipto Eucalyptus globulus L., pino Pinus sp L., Ciprés Cupressus sempervirens L., Aliso Alnus jorullensis H., Arrayan Myrcianthes leucoxyla O.; y frutales perennes como: Tomate de árbol Cyphomandra betacea C., Capulí Prunus serotina K., Reina Claudia Prunus sp L. y Chilacuán Carica gouditiana $\mathrm{L}$.

En la variable árbol, se evidenció que la mayoría de estudiantes conoce la importancia de éstos; sin embargo, manifiestan que por ser unidad agrícola familiar (UAF) menor a una hectárea, no es posible su inclusión dentro de sus parcelas; aunque después del proceso de capacitación y en la construcción del mapa visión de futuro (sueño), incluyen este componente en forma de arreglos agroforestales, lo que se convierte en un indicador de la incidencia que tuvieron los procesos de capacitación y actividades lúdicas en la aprehensión del conocimiento en este campo.

Lo anterior concuerda con los planteado por Padilla (1995), quien infiere que en la región Andina, las parcelas con árboles o arbustos asociados con cultivos, pastos y animales, no han sido instaladas bajo concepción agroforestal, pero funcionan y cumplen sus roles como tal; éstas, pertenecen a campesinos que han heredado la parcela y mantienen el conocimiento que les fue trasmitido de sus antepasados. Hay conciencia que es necesario manejarlas, pero se necesita conocer las tecnologías de manejo empezando por las propias, las que se practican en los Andes, las qué sean familiares y estén de acuerdo con la cultura agrocéntrica Andina, las que están siendo aplicadas en forma espontánea por los campesinos y aquellas que se conducen con apoyo de instituciones especializadas.

En el análisis de correspondencia múltiple ACM, se pudo observar que predominó el conocimiento sobre la importancia de los árboles (V3= si saben, 252 estudiantes) y la necesidad de implementar árboles en sus predios $(\mathrm{V} 4=\mathrm{si}$, 252 estudiantes); lo cual, puede ser atribuido al entorno rural donde la educación ambiental ha tenido un fuerte énfasis en la conservación de los recursos naturales, en donde la flora asume un papel preponderante; sin embargo, debido al nulo conocimiento del término agroforestería y sus implicaciones, fue necesario buscar estrategias para fortalecer éste en el campo de los SAF. Burbano (2013), plantea la importancia de la educación en la modalidad agropecuaria para la sostenibilidad a nivel de finca y región y para la recuperación de conocimientos y saberes propios de la sociedad nariñense.

En la figura 1 - B, se observa el bajo conocimiento agroforestal; por lo cual, los estudiantes manifiestan la importancia de abordar desde la infancia temas enfocados en la conservación de los recursos naturales y los beneficios de implementar sistemas agroforestales en sus fincas. Sentandreu (2012), plantea que en la pre-adolescencia (11-14 años), el individuo puede identificarse con su educador, ya sea padres, profesores, entre otros, buscando modelos a imitar, en relación a la conducta o toma de decisiones; por tanto, los medios audiovisuales y juegos utilizados en el proceso de capacitación, despertaron el interés de los estudiantes por aplicar abonos orgánicos, incluir el componente arbóreo e iniciar actividades de manejo de residuos. 
Piaget (2006), plantea que el pensamiento concreto, se desarrolla a partir de los 7 años, pero demuestra la incapacidad de generar sus propias hipótesis y de analizar cada circunstancia; por eso, es recomendable que todo lo que se pretenda enseñar, esté vinculado con su propia experiencia, es por ello que el SEAf está diseñado como una herramienta útil para la aprehensión del conocimiento agroforestal.

En relación a los abonos orgánicos y su importancia, tan solo un $27 \%$, de los estudiantes tenían un conocimiento previo (Figura 1B), en consecuencia, son poco usados en los sistemas productivos. El ACM, arrojó respuestas predominantes negativas (V5= no sabe, 169 estudiantes) por su preferencia a los insumos químicos; expresado en los beneficios de los fertilizantes, fungicidas e insecticidas; lo que conduce a cuestionar sobre la necesidad de explorar otro tipo de opciones más amigables con el ambiente, ya que en muchas fincas las áreas de producción son tan pequeñas que fácilmente podrían realizar un manejo agroecológico; lo cual, puede obedecer a la falta de presencia institucional que ofrezca capacitación y ejecución de programas agroecológicos; a pesar de que el Ministerio de Agricultura y Desarrollo Rural ofrece este tipo de servicios a través de sus programas; que reconocen su gran potencial para impulsar la productividad, promover la tecnificación del sector primario y fortalecer la articulación del país al mercado global; que dinamiza y gestiona el Plan Nacional de Desarrollo 2010 - 2014 "Prosperidad para todos" (Ministerio de Agricultura y desarrollo rural, 2013).

El $5 \%$ de los encuestados, demostró conocimiento sobre sostenibilidad y ninguno sobre agroforestería; datos que evidencian las necesidades de capacitación y orientación en temáticas relacionadas con recuperación y conservación de recursos naturales; y su relación con el desarrollo sustentable.
Al analizar el ACM de conocimientos sobre agroforestería (V8), sostenibilidad (V9) y sistemas agroforestales (V10), predomina el desconocimiento (256 estudiantes equivalentes al $91 \%$ ), lo cual puede obedecer a la carencia de formación integral por parte de las instituciones educativas; en donde los procesos de educación ambiental se orientan a la conservación de los recursos naturales en forma aislada de los sistemas productivos, por tanto, se deben articular los proyectos ambientales escolares PRAE, con los proyectos ciudadanos de educación ambiental - PROCEDA; acordes al contexto, a los medios de vida y a las tendencias de capacitación y orientación profesional, articulados a los planes educativos institucionales - PEI.

Según la FAO (2008), la implementación de programas de educación rural deben tener una formación académica coherente con sus modos de vida, creencias, saberes y prácticas cotidianas; ejemplo de ello, es el modelo pedagógicos "Escuela Nueva", programa de desarrollo rural integrado DRI, plan de fomento educativo para áreas rurales de municipios pequeños y escuelas urbano-marginales, proyecto de educación rural PER, entre otros.

Diagnóstico de gustos y preferencias. El ACM sobre los gustos y preferencias, mostró que los colores preferidos (V14) son el verde (126 estudiantes) y el azul (64 estudiantes); los personajes favoritos (V17) son los encontrados en la era de hielo (89 estudiantes) y el gato con botas (47 estudiantes); el paisaje preferido (V19) es el bosque (89 estudiantes) y las actividades que más les gustan son las competitivas (263 estudiantes), al aire libre (263 estudiantes) y grupales (257 estudiantes).

Durante las diferentes fases de desarrollo de un software es importante la obtención de información relevante, Galeano (2008), infiere que debe permitir diseñar una interfaz cercana a sus capacidades físicas cognitivas a sus 
gustos y expectativas, convirtiendo al usuario en coocreador; lo que se conoce como Diseño Centrado en el Usuario- DCU. Antón (2003), argumenta que es necesario definir perfiles de usuario (donde se detallan datos de preferencias de contenido, consumo, entorno) al momento de trabajar con sistemas digitales que permiten una personalización del producto y por ende una maximización de los recursos en oferta y demanda.

Análisis de los valores propios. El análisis del histograma de valores propios, permitió identificar que los cinco primeros factores explican en conjunto el $34.27 \%$ de la variabilidad; entre los que sobresalen como el primer factor, los colores preferidos, explicando el 7\% de la variabilidad. El segundo, tercero, cuarto y quinto factor (personaje preferido, paisaje favorito, juegos y área preferida) explican el $6,4 \%, 4,99 \%, 4,61 \%$ y $4,46 \%$, respectivamente.

Falguera (2010), al estudiar el comportamiento del individuo como consumidor de algún bien o servicio, plantea que al conocer sus necesidades y hacer el producto más aceptable, se llega a repercutir en su voluntad, por tanto, la aplicación del SEAf, permitió influir en los procesos de apropiación del conocimiento agroforestal, señalado principalmente por la aceptación de esta TIC por parte de la totalidad de los estudiantes involucrados.

Análisis de contribuciones de las variables. Se pudo establecer que las variables que más contribuyeron a la conformación de los factores fueron: existencia de árboles (V2=10,8), materia preferida (V15=10,1), existencia de sistemas productivos $(\mathrm{V} 1=9,9)$ y participación en las labores del predio $(\mathrm{V} 5=8,9)$; variables relacionadas en su mayoría con el entorno de los estudiantes (sistemas productivos).

Cortes y García (2009), afirman que en el desarrollo del niño y/o adolescente influyen los espacios físicos y las situaciones sociales; así mismo, Carvajal (2011), plantea que el entorno forma parte del sentido comunitario, reflejándose en la preocupación del individuo por éste y su participación para mejorarlo. En este sentido, el SEAf, le permite al usuario repensar su territorio y en las formas de actuar frente a él, en tanto, lo orienta a conocer buenas prácticas agrícolas para una posible adopción de sistemas agroforestales, como una opción de desarrollo local.

Diseño del software educativo agroforestal. Para el diseño del software, fue necesario el conocimiento previo agroforestal y de sostenibilidad. El 100\% de los encuestados manifestó no tenerlo y alrededor del $75 \%$ no conocía sobre temas afines; por tanto, se tomó la decisión de adelantar procesos de capacitación participativa sobre agroforestería y recursos naturales, en: sistema productivo agrícola, agricultura, abonos orgánicos, producción, erosión, vulnerabilidad, monocultivo, deforestación, reforestación, amenaza, sostenibilidad, agroforestería.

El proceso de capacitación permitió sensibilizar y captar la atención de los estudiantes hacia la importancia de los recursos naturales y el buen manejo de prácticas agroecológicas; además de contribuir a la identificación de los elementos requeridos para el diseño del SEAf (contenido, personajes, imágenes y juegos); en este particular, Sares (2010) opinan que el estudiante se puede involucrar con todo el empeño en algún proyecto, si juega un papel significativo en la elección y planificación del mismo al ver reflejadas sus motivaciones, gustos y preferencias.

En el diseño participativo del SEAf, se observó el interés y mejoramiento en el conocimiento agroforestal, al plantear la inclusión del componente leñoso perenne dentro de monocultivos y potreros desnudos, en una distribución espacial y temporal, evidenciándose, la importancia de capacitación previa por medio de videos y juegos; así como su motivación, al ser considerados 
participantes activos para su diseño. Skinner y Gonzás (2007), plantean que el aprendizaje es significativo cuando existe motivación, el "querer aprender", a través de juegos, actividades artísticas, videos, entre otros; además, Villamizar (2007), infiere que la motivación, requiere de conocimientos previos, la inteligencia y la experiencia.

Dentro del diseño del SEAf, se cuenta con las siguientes ventanas interactivas:

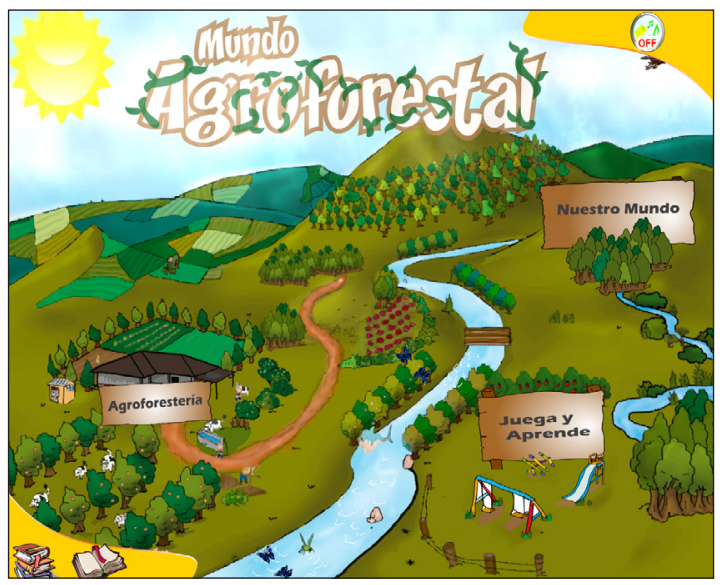

Figura 2. Menú principal del Software Educativo "Mundo Agroforestal"

Diccionario ambiental: Tiene los conceptos generales sobre recursos naturales y sistemas productivos agropecuarios, que introduce al estudiante en el conocimiento agroforestal (Fig. 3).

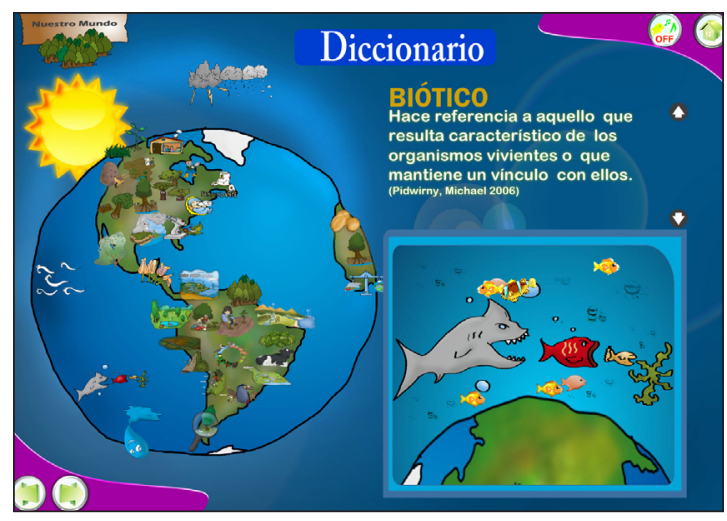

Figura 3. Ventana Diccionario del Software Educativo "Mundo Agroforestal"
Agroforestería:Abordasudefinición,clasificación y potencialidades (Fig. 4).

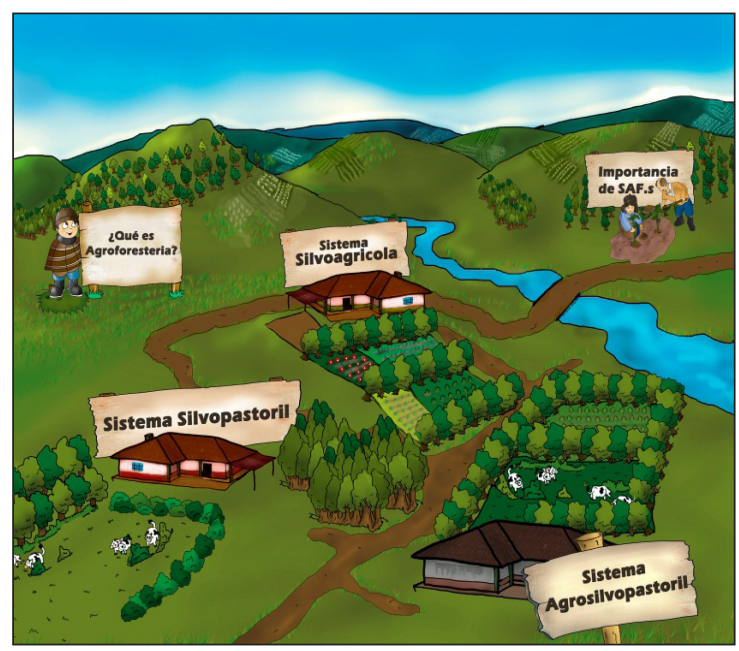

Figura 4. Ventana Agroforestería del Software Educativo "Mundo Agroforestal"

Juegos: Tienen el propósito de reforzar los conocimientos adquiridos por medio de cuatro juegos (Fig. 5): 1. "ENLÁZATE": permite relacionar la imagen o definición con la palabra que le corresponda. 2. "NARANJO DE RECUERDOS": el objetivo del juego, es potencializar la memoria, a través de la retención de imágenes y formación de parejas. 3. "DICHO Y HECHO": pretende inducir al estudiante a la reflexión, con respecto a los aspectos claves en el conocimiento sobre recursos naturales y agroforestería por medio de la ordenación de frases. 4. "TRIVIAMBIENTE": su objetivo es reforzar los conceptos encontrados en el diccionario. Los participantes deben responder las preguntas, entre cuatro opciones de respuesta.

Se incluyó el juego dentro de la presente investigación, teniendo en cuenta que el desempeño de los estudiantes mejoró a medida que se los involucraba dentro de actividades lúdicas, cabe resaltar que fueron ellos quienes crearon los juegos incluidos a través de la socialización de los mismos $\mathrm{y}$, determinar la pertinencia de éstos dentro del software. 


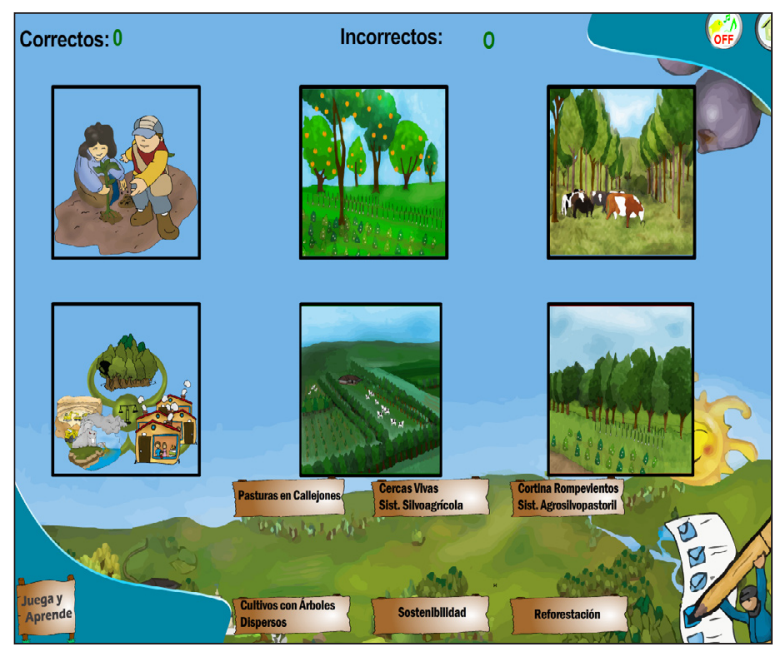

Figura 5. Ventana Juegos del Software Educativo "Mundo Agroforestal"

Leonel et al. (2013), plantean que es necesaria la implementación de herramientas didácticas innovadoras, que permitan o faciliten la construcción de individuos reflexivos, críticos y autónomos; lo que conlleva a su vez, a la generación de un enfoque pedagógico con la lúdica, como herramienta facilitadora de los procesos de educación, en este caso en el ámbito ambiental; Fandos et al. (2002), afirman que una estrategia didáctica se ve representada en la lúdica a través del juego, vista como una actividad libre, placentera y voluntaria; Leonel et al. (2013), infieren que "no se puede dar una definición única del juego; sin embargo, se puede asumir como un sistema que permite el desarrollo de los individuos, que al seguir unas reglas predeterminadas, pueden llegar a cumplir con los objetivos propuestos por el juego, en donde las acciones, estrategias y vivencias previas de los jugadores, son los elementos diferenciadores para la diversión y consolidación de habilidades de los seres humanos".

\section{Evaluación delSoftwareEducativo Agroforestal} SEAf "Mundo Agroforestal". En la evaluación Alfa, se evidenció el interés y la disponibilidad de trabajar con este tipo de herramientas; además, los estudiantes se apropiaron del resultado final al ver reflejado su trabajo en el software. En la evaluación Beta, se observó la aceptación del
SEAf en todas las variables evaluadas (diseño, facilidad de navegación, pertinencia de colores, temáticas abordadas e ilustraciones), con un promedio de $94 \%$ de personas que calificaron cada variable como altamente adecuada, 6\% moderadamente adecuada y $0 \%$ no adecuada.

El proceso investigativo evaluado, resultó ser altamente aceptado por los usuarios, posiblemente por lo planteado por Caliva (2009), quien afirma que durante su vida el ser humano anhela alcanzar objetivos y metas que les permitan integrarse a la sociedad, esto lleva a educarse de una forma permanente, activa y organizada durante toda su vida, implementando diferentes estrategias de aprendizaje; para el caso agroforestal, el SEAf, se convierte en un instrumento mediador para acceder al aprendizaje. Después de que el estudiante haya explorado espontáneamente este instrumento y encontrado una relación con el objetivo del mismo, se continúa el proceso de aprendizaje a través de la decodificación lógica del material (representación mental del concepto); para ello, es necesaria la discusión, confrontación de hipótesis, procesos de exposición de las elaboraciones que han construido los estudiantes y de argumentación en los que se confrontan hipótesis y se accede a acuerdos sustentados.

Lo anterior, de acuerdo a Vygotsky (2008), se denomina "internacionalización" y se define como el momento en que el saber cultural, externo al sujeto, pasa a ser una realidad mental apropiada, como producto de la interacción con otros; es decir una representación mental construida por el sujeto de aprendizaje.

Teniendo en cuenta las características presentes en el software educativo agroforestal y el resultado de la evaluación - altamente adecuado, se puede inferir que el software queda validado y disponible para ser utilizado por el público objetivo (estudiantes de secundaria pertenecientes a instituciones rurales), de uso libre para cualquier persona y con propiedad intelectual de los autores de éste y de la Universidad de Nariño. 


\section{CONCLUSIONES}

La generación de espacios participativos para la investigación, la comunicación, el diálogo de saberes y el fomento de la creatividad, transversalizados por la lúdica; conllevan a incrementar la motivación del estudiante, quien se hace participe en la contribución de tecnologías de la información y comunicación, como lo es el SEAf, siendo este socialmente valorado.

El software educativo "Mundo Agroforestal", cuyas características demuestran armonía entre interfaces, innovación en el ámbito educativo, facilitador de la información, interactivo y la inclusión del juego como estrategia pedagógica, se consolida como una herramienta educativa motivante y generadora de interés en la trasmisión del conocimiento agroforestal.

\section{BIBLIOGRAFÍA}

ANTÓN, C. 2003. Desarrollo de un Sistema de Gestión de Perfiles de Usuario para la Personalización en el Acceso a Contenidos Digitales. Universidad Politécnica de Madrid. Departamento de Ingeniería de Sistemas Telemáticos. 25 p.

AUSUBEL, D.; NOVAK, J. y HANESIAN, H. 1983. Psicología educativa. Un punto de vista cognitivo. Segunda edición. Trillas. México, p.20

BURBANO, N. 2013. Educación para el progreso del estudiante del sector rural en Nariño. Revista Entramado. Universidad libre de Cali. 9 (1):112 p.

CALIVA, J. 2009. Manual del capacitador para el facilitador. Centro de liderazgo en agricultura. Instituto Interamericano de Cooperación para la Agricultura (IICA), San José de Costa Rica. 102 p.

CARVAJAL, A. 2011. Apuntes sobre el desarrollo comunitario. Primera Edición, Eumed.net, Universidad de Málaga, España. 75 p.

COLCIENCIAS. 2012. Apropiación social del conocimiento. Sistema nacional de ciencia, tecnología e innovación. En: www.colciencias.gov.co/programa_estrategia/apropiaci-n-social-del-conocimiento; Consulta: marzo, 2014.

CORTES M. y GARCIA E. 2009. La influencia del contexto sociocultural en el desarrollo cognitivo de niños y adolescentes. Tesis de especialización de Sicóloga, Facultad de Humanidades, Escuela Normal de Especialización, Sinaloa, México DF. $77 \mathrm{p}$.

DEPARTAMENTO ADMINISTRATIVO NACIONAL DE ESTADÍSTICA (DANE). 2010. Censo General 2005, boletín 13/09/2010, Perfil Nariño. En: https://www.dane.gov.co/files/censo2005/PERFIL_PDF_CG2005/52000T7T000.PDF. 6 p.; consulta: junio, 2014.

DEPARTAMENTO ADMINISTRATIVO NACIONAL DE ESTADÍSTICA (DANE). 2012. Plan de Desarrollo Departamental (2012 - 2015): Nariño mejor. República de Colombia. Gobernación de Nariño. En:http://www.narino-narino.gov.co/ apc-aa-files/3834613362656534313935363465653 6/nariodiagnostico verticalok-_29_05_2012.pdf; consulta: junio, 2014.

FALGUERA, L. 2010. Importancia de conocer la conducta del consumidor. Revista ESHOB. 11(1):5-11.

FALS, O. 2008. La investigación acción participativa. Revista Espacio Abierto. 17(2): 4-10.

FANDOS, M.; JIMÉNEZ, J. y PIO, A. 2002. Estrategias didácticas en el uso de las tecnologías de la información y la comunicación. Revista Acción Pedagógica. 11(1): 28-39.

GALEANO, R. 2008. Diseño centrado en el usuario (articulo de investigación académica, científica y tecnológica). Revista Q, 2(4): 12-14. En: http:// revistaq.upb.edu.co; consulta: agosto, 2013.

GALVIS, E.; MARTINEZ B.; OLIVEROS, L. y OLIVEROS, M. 2012. Las TIC: Herramientas motivadoras para la apropiación de la lecto-escritura a través de textos. Trabajo de grado de Licenciatura en Educación Básica con énfasis en Tecnología 
Informática. Facultad de Educación, Universidad Cooperativa de Colombia, sede el Bagre, Antioquia. $59 \mathrm{p}$.

HONORABLE CONCEJO MUNICIPAL DE PASTO. 2012. Acuerdo No. 008 de mayo 31, por el cual se aprueba el plan de desarrollo del municipio de Pasto 2012 - 2015. "Transformación productiva". Alcaldía de Pasto. Colombia. 210 p.

LAVADO, A. 2011. Las TIC como medio para perfeccionar la dirección del proceso de enseñanza. Revista IPLAC, Publicación Latinoamericana y Caribeña de Educación. 4 (3): 2-5.

LEONEL, H.; LUNA, G. y RIVAS, H. 2013. El juego como herramienta para la educación ambiental, pp 222 - 230. En: Universidad Mariana (compilador). Agua para la vida. Primera edición. Publicaciones UNIMAR. Colombia, 456 p.

MARQUES P. 2001. Los procesos de enseñanza y aprendizaje. La motivación. Universidad de Oviedo. España. 25 - 28 p.

MINISTERIO DE AGRICULTURA Y DESARROLLO RURAL. 2013. Estrategia de cooperación internacional del sector agropecuario 2013 - 2015, Colombia. En: https://www.minagricultura.gov. co/ministerio/Documents/Estrategia-de-Cooperacion-Internacional-del-Sector-Agropecuario-2013-2015.pdf. 34 p; consulta: agosto, 2014.

MINISTERIO DE TECNOLOGÍAS DE LA INFORMACIÓNY LAS COMUNICACIONES DE LA REP. DE COLOMBIA. 2008. En: http:/ / www.mintic.gov. co/; consulta: junio, 2013.

ORGANIZACIÓN DE LAS NACIONES UNIDAS PARA LA AGRICULTURA Y LA ALIMENTACION (FAO). 2008. El estado mundial de la agricultura y la alimentación. Colección FAO: Agricultura No 27. Roma. $55 \mathrm{p}$.

PADILLA, S. 1995. Manejo forestal andino. Proyecto FAO-Holanda "Desarrollo Forestal Participativo en los Andes", E.P. Centro de Impresión. Ecuador. $297 \mathrm{p}$.
PIAGET, J. 2006. Seis estudios de Psicología. Primera edición. Labor S. A. Barcelona, España. 199 p.

ROBEY, D. 2007. TIC y cambios organizativos. Edutecne. Oxford. $39 \mathrm{p}$.

SARES, O. 2010. Aplicación de estrategias metodológicas de enfoque constructivista en la motivación hacia el aprendizaje en los niños y niñas de 5 a 6 años $\mathrm{d}$ las escuelas Ing. Jorge Alex Serrano, Oscar Efrén Reyes, Luís Aguilar Marín, Charbel Badul. Tesis de pregrado de licenciado en ciencias de la Educación. Facultad de Ciencias Sociales. Universidad Técnica de Machala. Ecuador. 158 p.

SKINNER, C y GONZAS, M. 2007. Dimensiones espaciales de la realidad social. Eumedent. España. $86 \mathrm{p}$.

SECRETARÍA EDUCACIÓN MUNICIPAL DE PASTO. 2012. Plan de desarrollo educativo 20122015 “Educación con calidad y equidad para la transformación social". $127 \mathrm{p}$.

SENTANDREU, J. 2012. Adolescentes. Para la formación integral. Ediciones Sti. Ganduxer.Primera edición. Barcelona. 36 p.

VIGOTSKY, L. 2008. Pensamiento y Lenguaje (Teoría del desarrollo de las funciones psíquicas). México, Ediciones Quinto Sol. 191 p.

WORLD AGROFORESTRY CENTRE, 2011. Transformando vidas y paisajes. Disclamer. CIFOR, CIAT, ICRAF. 77-81 p.

VILLAMIZAR, L. 2007. Estrategias de formación de profesores universitarios para el uso de las tecnologías de información y Comunicaciones (TIC) a partir del sistema de aprendizaje let me learn: dos estudios de caso. Tesis doctorado interuniversitario en tecnología educativa, Universidad Rovira I Virgili, Facultad de Ciencias de la Educación y psicología, departamento de Pedagogía. Terragona - España. 195 p. 